\title{
A Visualization Technique of a Music Emotion Represented in Dimensional Approach on Infinity Mirror Based LED Wall
}

\author{
Van Loi Nguyen, Xu Bin, Li Hui, Lin Bin, Donglim Kim and Younghwan Lim \\ Department of Media, Soongsil University \\ vanloiktkt@yahoo.com,xubinyj@126.com,lihui0928@gmail.com, \\ linbin98@gmail.com,dlkim@ssu.ac.kr,yhlim@ssu.ac.kr
}

\begin{abstract}
In this paper, we propose a visualization method of a music emotion on LED wall. Emotion in music is recognized by a suggested algorithm using a dimensional approach. The algorithm overcomes some shortcoming of Thayer's model in detecting emotion in a one-second music segment. Moreover, IRI color model is combined with Thayer's model to determine LED light colors corresponding to 36 different music emotions. They are visualized through colors and animations on LED wall. The accuracy of music emotion visualization achieved to over $60 \%$.
\end{abstract}

Keywords: music feature extraction, music emotion recognition, music emotion variation detection, music emotion visualization, LED wall

\section{Introduction}

Music plays an important role in our lives, even more so in the digital age. One of the most appealing functions of music that it can convey emotion and modulate a listener's mood. It is generally accepted that music cannot be composed, performed, or listened to without affection involvement. Music can lull a baby to sleep and bring a person to tears. Its effects are felt clear, but the methods using to touch our hearts are more elusive. How to help audiences perceive better the emotional content in music is a big question. Visualization techniques make a fitting environment show music emotion. Their capacity for flexibility, creativity, and multidimensionality facilitates the presentation of abstract data. In addition, feeling emotions is the primary motive of listening to music. Consequently, the music performed with a visualizer supports listeners to enhance the musical experience.

Recently, research in music emotion visualization has been very active with different media. For example, a music mood visualization tool named Mood cloud was developed by Laurier and Herrera [7]. While playing a song, the software calculates probabilities of five emotion categories: "happy", "relax", "aggressive", "sad", and "party". Each mood is presented by a colored bar graph with text, dynamically resized according to the emotion probability. Using photos for emotion visualization was performed by Chen, Weng, Jeng, and Chuang in 2008 [5]. They collect emotionally charged photographs, and pair these pictures with various music selections. Taylor, Boulanger and Torres utilized a virtual character for visualizing emotion in musical performance [8]. The character can show its simulated emotional response through visual animations generated in real-time and displayed on a life-sized stereoscopic screen. However, the media for visualizing music emotion in previous work are difficult to satisfy a large number of audiences in musical performance.

This research suggests an LED lighting control system that is a new medium to visualize music emotion through colors and animations. Because the light system plays an important role in musical performance. However, this system involves a lot of manual effort of experts to control it. Therefore, some colors and animation patterns on LED wall 
are irrelevantly displayed to the music emotion content. In the proposed system, colors and animations controlled based on music emotion will raise the effect of musical performance. The integration of different forms of media could evoke even more feelings and give a more touching presentation as long as they are synchronized in emotions. Listeners can perceive deep emotion from songs through both visual and auditory media. Figure 1 presents an overview of our system.

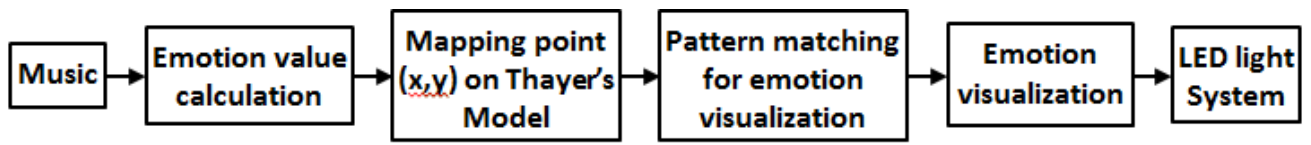

Figure 1. The Flowchart of Suggested System

The presented visualization system consists two main parts: a hardware system and a software controlling the light system based music emotion. Firstly, the music emotions are recognized by a proposed algorithm using a dimensional approach. Then LED light colors corresponding to 36 different music emotions are specified by combining the IRI color model with Thayer's model. Finally, animation patterns in accordance with music emotions are created and presented on the light system.

The rest of this paper is organized as follows. Section 2 shows emotion recognition and sampling in a single music. Matching emotions with colors is described in Section 3. Section 4 introduces color rendering on LED wall. Experimental results are presented in Section 5. Finally, Section 6 provides conclusions.

\section{Emotion Recognition and Sampling in a Single Music}

\subsection{Emotion Recognition}

Emotion visualization in music can be divided into two major stages: detecting emotion and visualizing emotion. Methods used in recognizing emotion can be grouped into two general approaches: categorical approach and dimensional approach. In categorical approach, emotions are grouped into basic emotion classes using machine learning techniques. This approach is rarely used in emotion visualization because of the limited number of emotions and emotion recognized in each music segment (often 30 seconds) or the whole song.

In this study, music emotion is detected by the dimensional approach. The emotion of one-second music segment is modeled as a point $(\mathrm{x}, \mathrm{y})$ on Thayer's model. In the model, emotions are distributed in a two-dimensional space, containing arousal and valence dimensions. The vertical axis represents arousal (or activation; energy and stimulation level) while valence (or pleasantness; positive and negative) is indicated on the horizontal axis. Each point of Thayer's model represents an emotion in the music section and calculated based on acoustic features. 5 music features selected that affect the music emotion consisting of tempo (BPM), dynamics (RMS), amplitude change (Roff off), brightness (Centroid), and noise (Zero Crossing). They are extracted by MATLAB and MIR (Music Information Retrieval) ToolBox [6].

Table 1 shows 5 music features extracted from a song called "Can't go on my own" that has sung by "Clazziquai". The mean value and the standard deviation value are also extracted by analyzing 160 songs from website "ollehmusic.com". 
Table 1. Features Extracted from Music Samples

\begin{tabular}{|l|l|l|l|l|l|}
\hline Data & BPM & RMS & Roff off & Centroid & Zero Crossing \\
\hline Extracted values & 90 & 1.44 & 1317.74 & 9663.87 & 3890.06 \\
\hline Average & 121.42 & 0.73 & 1081.29 & 6069.61 & 2638.64 \\
\hline Standard Deviation & 18.28 & 0.37 & 478.05 & 2490.39 & 958.67 \\
\hline
\end{tabular}

The units and range of the extracted feature values are different. So it is difficult to use the data combination of each element. Normal Distribution is used for calculating the probability of feature values based on the mean and standard deviation. Apache libraries were implemented in Java environment for calculating the probability values.

Music feature values need to be mapped on Thayer's model for detecting emotion. However, the influence of these values to Arousal on the $\mathrm{X}$ axis and Valence on the $\mathrm{Y}$ axis is different. We conducted experiments on 160 songs of the sample data to find the suitable weights of music feature values. Experimental results are shown in Table 2.

Table 2. The Weights of the Five Music Features

\begin{tabular}{|l|l|l|l|l|l|}
\hline & Bmp & RMS & Roff off & Centroid & Zero Crossing \\
\hline X (Variable) & $20 \%$ & $10 \%$ & $40 \%$ & $20 \%$ & $10 \%$ \\
\hline Y (Variable) & $40 \%$ & $10 \%$ & $10 \%$ & $20 \%$ & $20 \%$ \\
\hline
\end{tabular}

According to reasonable weights of the five music features in Table 2, the coordinates of point $\mathrm{P}(\mathrm{x}, \mathrm{y})$ are calculated as follows:

$x=0.2 *$ Bmp $+0.1 * R M S+0.4 *$ Roff off $+0.2 *$ Centroid $+0.1 *$ Zero Crossing

$y=0.4 *$ Bmp $+0.1 * R M S+0.1 *$ Roff off $+0.2 *$ Centroid $+0.2 *$ Zero Crossing

\subsection{Sampling in a Single Music}

After calculating the points $(\mathrm{x}, \mathrm{y})$, these points are mapped onto Thayer's model to determine emotion [2-3]. For music emotion visualization, emotion should be detected in each short-time segment. The length of a segment is often less than or equal to 16 seconds such as one second [1], ten seconds and 1/3 overlap [9]. However, there are some weaknesses when using Thayer's model to recognize the music emotion in each shorttime segment. The points near the center of Thayer's model have different emotions despite their distances are very close (small change of acoustic features) such as A1, A2, A3 in Figure 2. Conversely, the distance between A2 and A4 is so far but they have the same emotion.

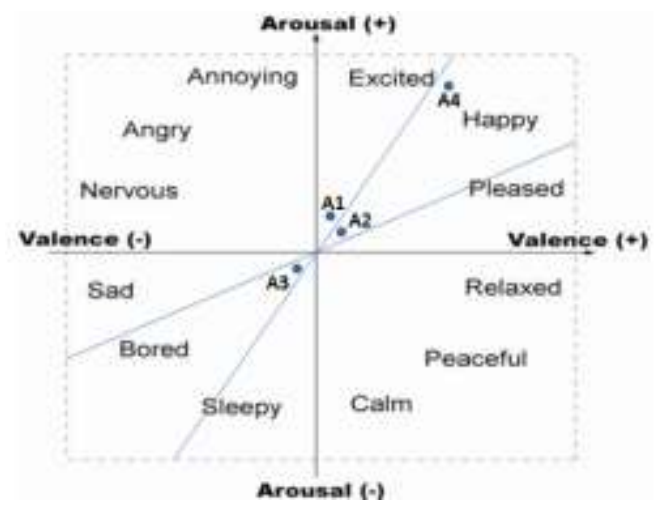

Figure 2. Thayer's Model for the Emotional Plane (Also named Russel's Model) 
We suggested an algorithm for music emotion variation detection. In this algorithm, emotion in two consecutive one-second music segments will be the same if there is little change in music feature values (in other words, the distance between two points in Thayer's model is less than a given constant named "d"). The algorithm is applied in a process named "sampling" which gathers successive one-second music segments with the same emotion to a music section. The sampling in a single music is illustrated in Figure 3.
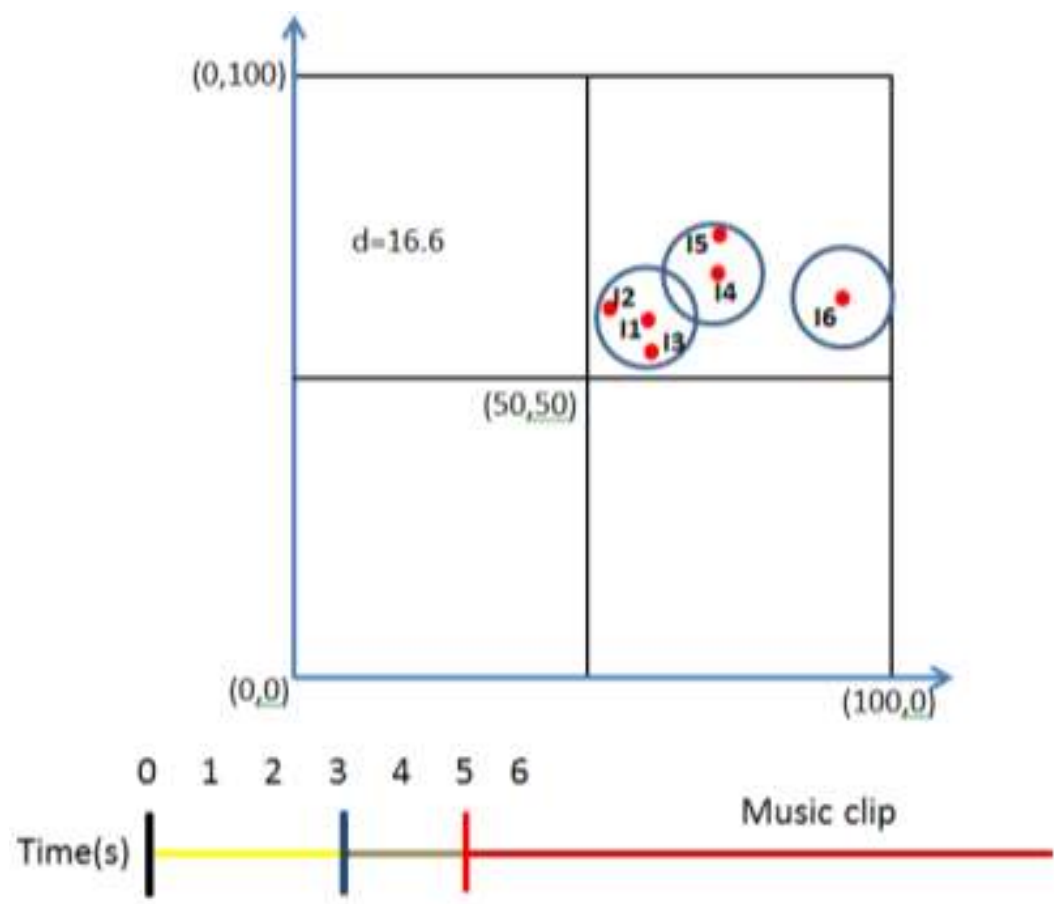

Figure 3. An Example of the Music Sampling Process

Start a new section, a circle is drawn with diameter $\mathrm{d}=16.6$ and center $\mathrm{I} 1$. I2 and I3 have the same emotion with I1 because they are in the first circle. I4 is outside of the first circle, so Section 1 ends with 3 one-second segments which have the same emotion. The second section starts with drawing a circle with center I4. It is clear that this section contains 2 one-second segments. The process is repeated until the end of the music clip.

In sampling process, the emotion of each section is calculated based on the center of the circles which are employed for matching colors. Thayer's model will be divided into 36 areas corresponding to 36 emotions recognized from music when the diameter of the circle is 16.6. Besides that, music does not need to be broken into one-second segments for visualization. The number of recognized emotions can be adjusted by the diameter of the circle. With $\mathrm{d}=16.6$, the 80 -second music 'Kurenai' was sampled into 8 sections. The result of the sampling process is shown in Figure 4.

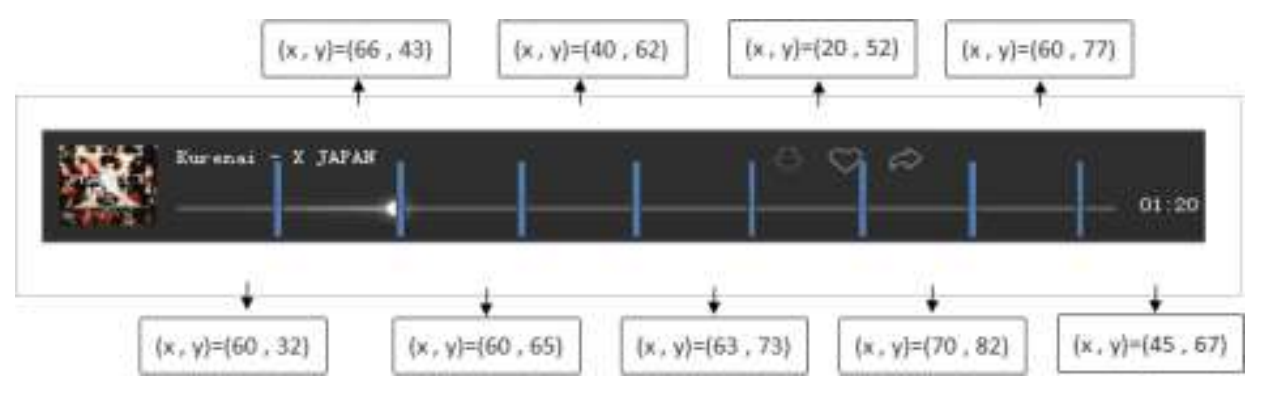

Figure 4. Sampling Music 'Kurenai' 


\section{Matching Emotions with Colors}

After detecting emotions in music, they are visualized through the colors on LED wall. To find the relationship between emotion and color, we rely on the correlation between Thayer's model and IRI color model published by Scale Research Institute. In IRI model, particular colors or color combinations are related and arranged with emotional adjectives [11-12].

Comparing Thayer's model and IRI model, we realize that if the IRI model is rotated 90 degrees clockwise, emotions in Thayer's model and affective adjectives in the IRI model will have the same meaning and position such as "Excited-Nimble", "Happy-Fun", "Pleased-Refreshing". Figure 5 illustrates the combination of 2 models.

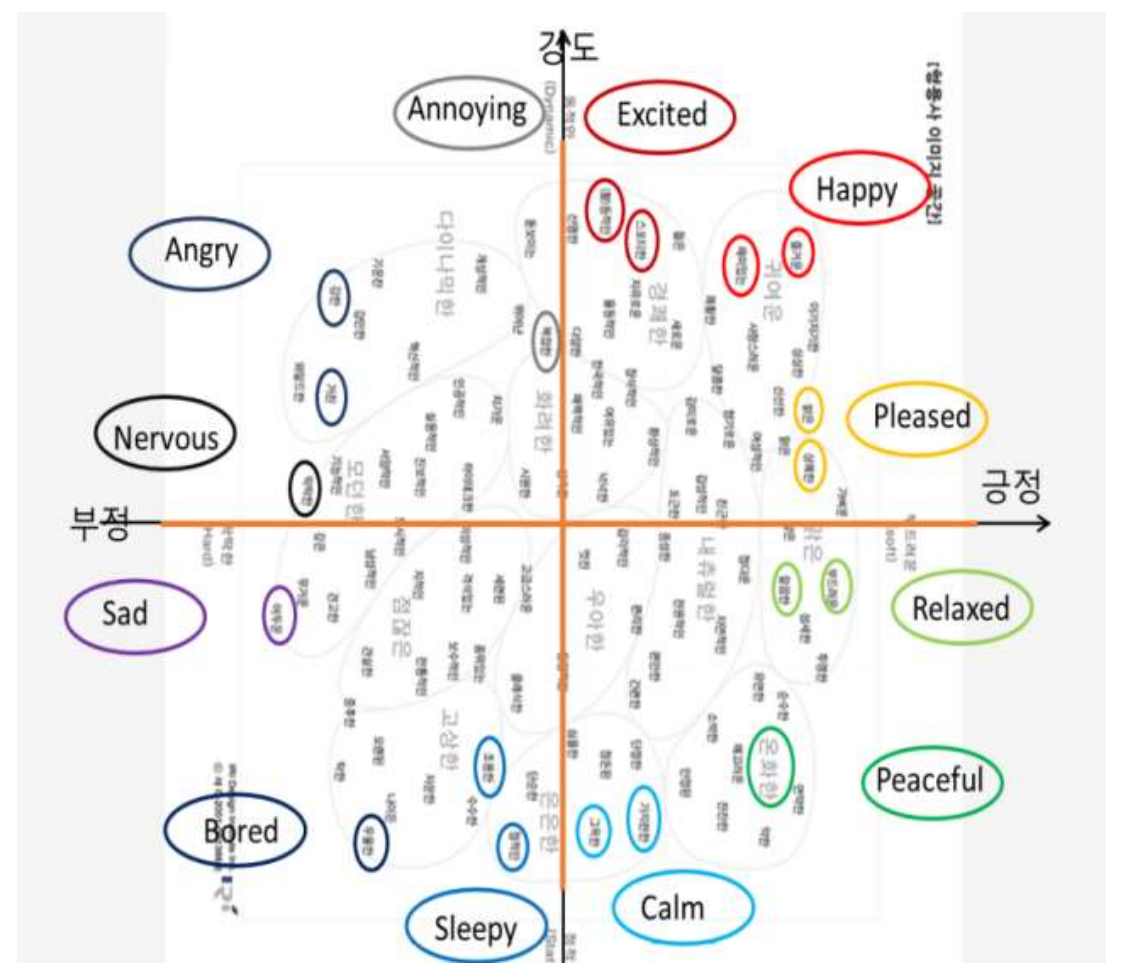

Figure 5. The Reunification of Thayer's Model and IRI Color Model

Thanks to the association between emotion model and color model, 36 areas of Thayer's model are mapped onto the IRI model to find appropriate colors with 2 following rules. Rule 1 is that one appearance including 3 colors is chosen, if it is the closest to the center of the area. In the last rule, one appearance can be only chosen one time. A list of 363 -color groups corresponding to 36 emotion areas is created based on the rules. Colors are determined based on music sections which are calculated from the process of sampling. These sections are represented by the center of the circles which are mapped into Thayer's model to specify the emotion sections.

\section{Color Rendering on LED Wall}

LED wall used the CIE 1931 color spaces which were created by the International Commission on Illumination (CIE) in 1931. However, a few of RGB values are incorrectly displayed on the LED light system. For example, the RGB $(32,30,30)$ is blue, but it is displayed as black on the LED light system as presented in Figure 6. 


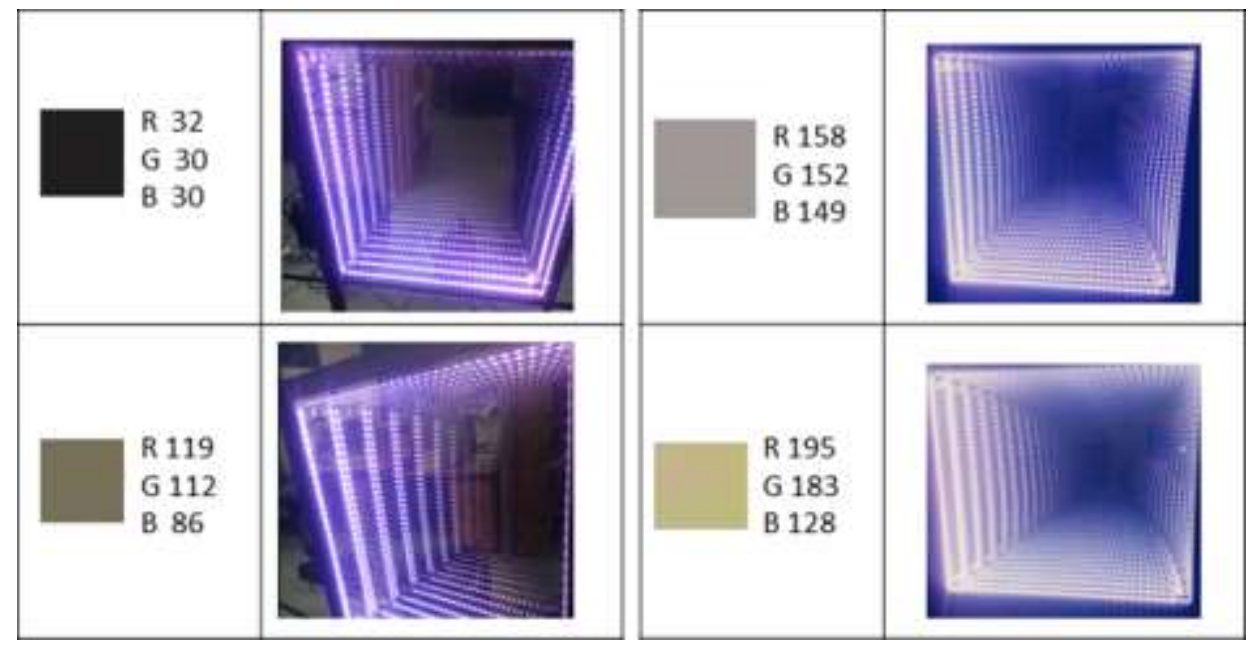

Figure 6. RGB Colors Displayed Incorrectly on LED Lighting

We proposed a method to replace these incorrectly-displayed colors. If an RGB color is not in the list of the CIE color palette. The 3-color group of each music section containing it will be only replaced by one color. The representative color is designated thanks to Emotions Color Wheel which can help visually group feelings as indicated in Figure 7 [13].
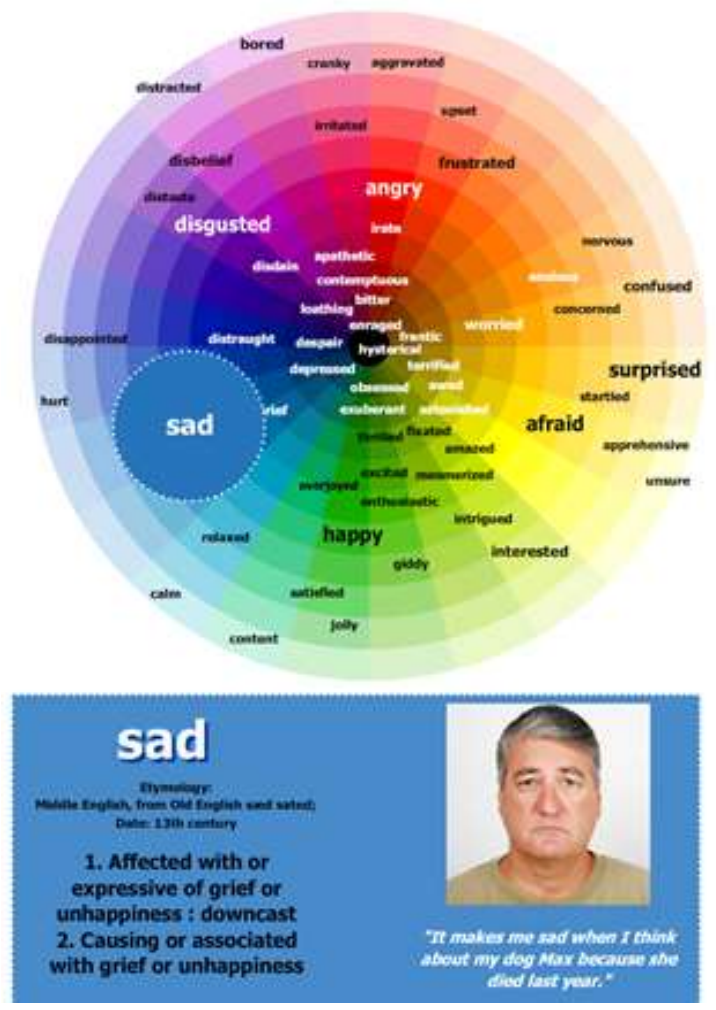

Figure 7. Do2Learn's Emotions Color Wheel

Emotions Color Wheel is a fascinating perspective on the influence of color on emotions, generating feelings by their usage. It is designed for educational purposes by Do2Learn. The circle is divided into colors to show some basic emotions. Figure 8 presents appropriate to 12 colors extracted from Emotion Color Wheel on Thayer' Model. 


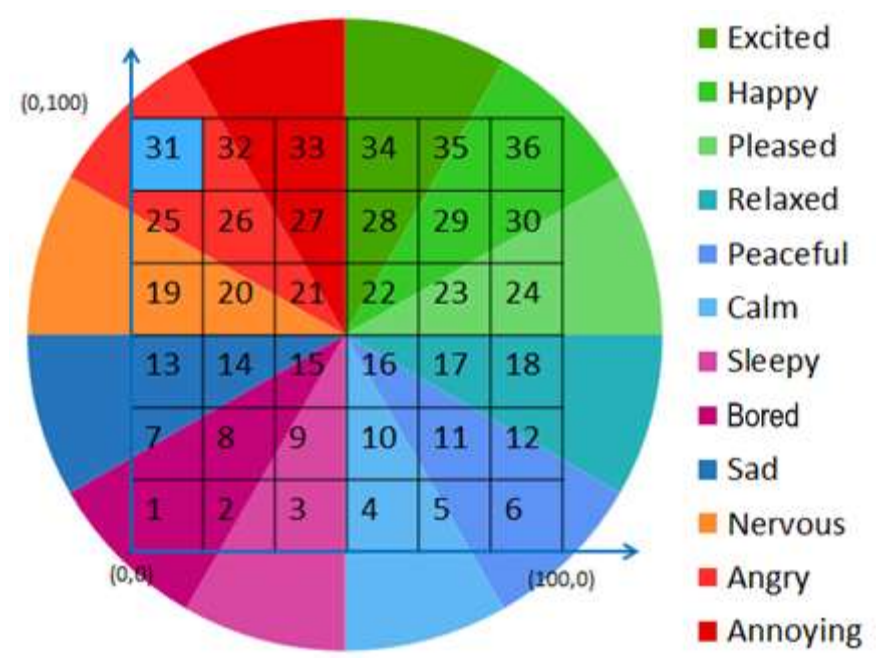

Figure 8. 12 Colors Represented in Thayer' Model

Colors in accordance with music emotion need to store for controlling the light system. In this work, they are stored in the XML file. For example, after calculating acoustic features of a music section, we get a point $(28,24)$ on Thayer's model. This point belongs to Section 10 which is used to find suitable colors.

\section{Experimental Results}

\subsection{Infinity Mirror Based LED Wall}

The LED WALL is the most important component of the emotional LED lighting control system which consists of 1 PC, 3 LED Moving Head Light, 3 LED Par 64, 1 screen and 1 LED wall. The LED WALL comprises 40 LED boxes (5 rows and 8 columns). The LED box consists of 4 LED Bar and an infinity mirror as shown in Figure 9.

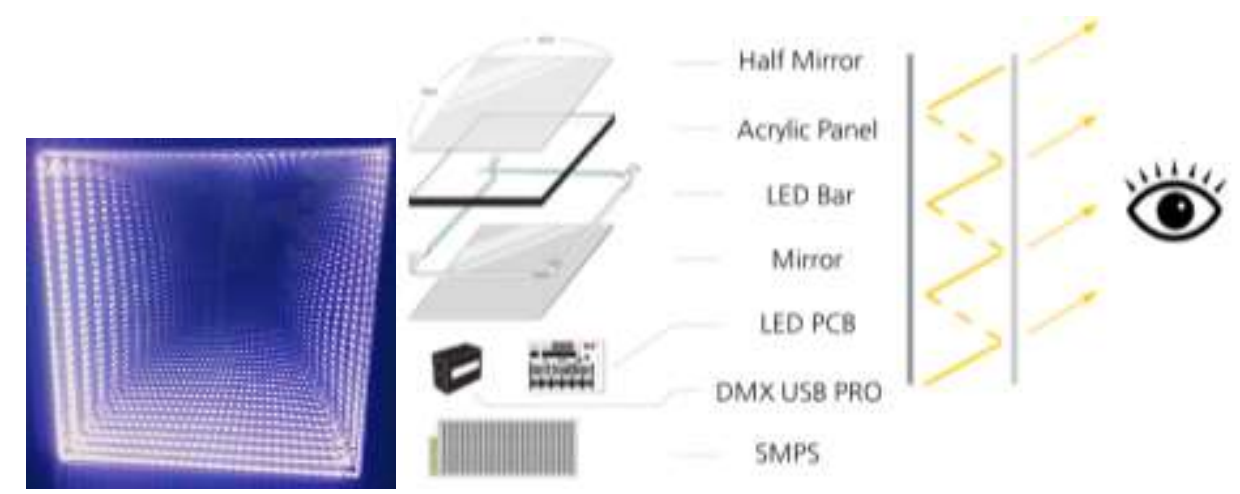

Figure 9. The Construction of Infinite Mirror

The infinity mirror is a set of mirrors, one fully reflective mirror and one "one-way mirror", set up so that the one-way or partially reflective mirror reflects an image back onto the fully reflective mirror, in a recursive manner, creating a series of smaller and smaller reflections that appear to recede into an infinite distance. 


\subsection{Visualizing Music Emotion}

The LED wall can be automatically controlled based on recognized emotion by the main program. It is written in program language VVVV (V4) to read data from XML file and generate visuals through colors and animations on LED wall.

Colors need to be displayed on LED wall in accordance with music emotion. Firstly, one program is written in Matlab for detecting emotion in music. Then it calculates 3color groups appropriate for emotions in music sections. Finally, an XML file is generated to store these 3-color groups. The main program reads color data from the XML file by a module named "read file". Colors displayed on LED wall is shown in Figure 10.
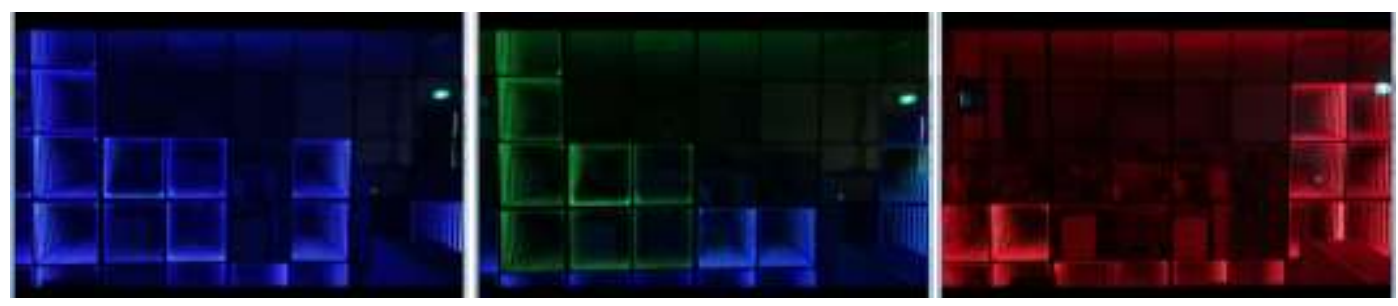

Figure 10. Colors Displayed on LED Wall

With the purpose to increase the fascination of LED wall, we created 12 animations on LED wall for 12 music emotions. Firstly, an LED box was created in V4 as shown in Figure 11. Then the animations were created on LED wall based on the state ON/OFF of LED box. The state of LED boxes is controlled according to the speed and time as well as under a specific rule for each emotion.

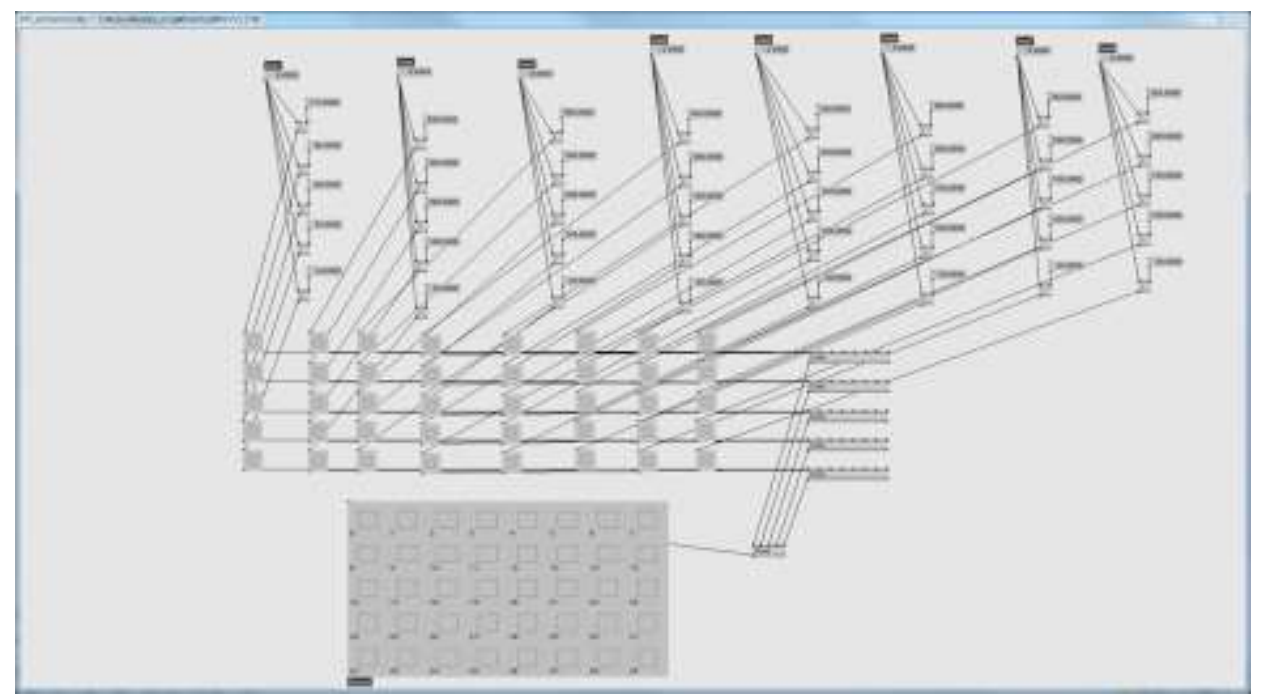

Figure 11. Creating an LED Box in V4

It is necessary to find the animations on LED wall matching with music emotion. We created 3 animations each emotion. A survey was conducted on 50 people who filled out the visualization questionnaire, in which they chose an animation that according to them should be used to visualize a particular music emotion. The animation selected with the highest percentage in the survey will be shown on the LED wall. 


\subsection{System Verification}

After installing the devices similar to a small stage, a survey was conducted in order to verify the consensus in the performance of the light system. 12 30-second music clips with 12 different emotions were collected from All Music Guide (AMG) [10]. The labels of these music clips are manually assigned by musical experts. 80 people listened to the music clips in the atmosphere of music emotion visualization by the light system. Then subjects gave feedback about their feeling for the light system in each music clip. The survey results are indicated in Table 3.

Table 3. The Survey Results of the Light System

\begin{tabular}{|c|c|c|c|c|c|}
\hline \begin{tabular}{|l|} 
Emotion \\
\end{tabular} & Accuracy & Pie chart & Emotion & Accuracy & Pie chart \\
\hline Excited & $65 \%$ & & Sleepy & $63.75 \%$ & \\
\hline Happy & $66.25 \%$ & & Bored & $63.75 \%$ & \\
\hline Pleased & $62.5 \%$ & & $\mathrm{Sad}$ & $66.25 \%$ & \\
\hline Relaxed & $58.75 \%$ & & Nervous & $58.75 \%$ & \\
\hline Peaceful & $60 \%$ & & Angry & $65 \%$ & \\
\hline Calm & $61.25 \%$ & & Annoying & $58.75 \%$ & \\
\hline
\end{tabular}


In our study, recognizing music emotion is based on emotion variation detection. Two successive one-second music segments will be the same emotion if the distance between the points representing them on Thayer's model is small (probably located in two different emotional areas). Therefore, the accuracy of the LED light system for each emotion is defined as a total of the accuracy of 3 consecutive emotions. For example, the accuracy of the music piece with emotion "Excited" is $65 \%(38.75 \%+17.5 \%+8.75 \%)$. The average accuracy of the suggested system achieved at $62.5 \%$. The rate is quite satisfied because recognizing and visualizing of music emotion are difficult tasks due to the subjectivity of emotional perception. Music perception is intrinsically subjective and is under the influence of many factors like cultural background, age, gender, personality, training, and so on [4].

\section{Conclusion}

We have described a new strategy for music emotion visualization. To achieve this goal, an emotional LED system was implemented to effectively enrich music listening experience. So musical performance is more effective by music emotion visualization through colors and animations on LED wall. The system can be automatically controlled according to recognized emotion.

In this study, we proposed an emotion recognition algorithm using dimensional approach. It improves the weaknesses of Thayer' model in recognizing music emotion in a one-second music segment. Besides that, we found out the relationship between emotion and color based on the association of Thayer emotion model and IRI color model. A survey was conducted to choose the best animation pattern for each music emotion. Another survey showed the suitability between music emotion and visualizing it on the light system. User evaluation shows that light system is more impressive and affecting. The accuracy of music emotion visualization reached $62.5 \%$. We believe that the system presents a novel way to visualize music emotion in musical performance and understand the emotive capacity of music.

\section{Acknowledgments}

This paper is a revised and expanded version of a paper entitled "A Real-time Method for Recognition and Visualization of a Music Emotion on LED Light System" presented at the $4^{\text {th }}$ International Conference on Smart Media and Applications, Korea-Vietnam Friendship IT College, Danang, Vietnam, January 10-13, 2016.

\section{References}

[1] D. K. Mark, D. Clausi and M. Jernigan, "Modeling emotional content of music using system identification”, Systems, Man, and Cybernetics, Part B: Cybernetics, IEEE Transactions on, vol. 36, no. 3, (2005), pp. 588-599.

[2] A. R. James, "A circumplex model of affect", Journal of personality and social psychology, vol. 39, no. 6, (1980).

[3] E. T. Robert, "The biopsychology of mood and arousal", Oxford University Press, (1989).

[4] Y. Y. Hsuan and H. H. Chen, "Music emotion recognition", CRC Press, (2011).

[5] C. C. Han, M. F. Weng, S. K. Jeng and Y. Y. Chuang, "Emotion-based music visualization using photos", Advances in Multimedia Modeling, Springer Berlin Heidelberg, (2008), pp. 358-368.

[6] L. Olivier and P. Toiviainen, "A Matlab toolbox for musical feature extraction from audio", International Conference on Digital Audio Effects, (2007), pp. 237-244.

[7] L. Cyril and P. Herrera, "Mood cloud: A real-time music mood visualization too", Proceedings of the Computer Music Modeling and Retrieval, (2008).

[8] T. Robyn, P. Boulanger and D. Torres, "Visualizing emotion in musical performance using a virtual character", Smart Graphics, Springer Berlin Heidelberg, (2005), pp. 13-24.

[9] Y. Y. Hsuan, C. C. Liu, and H. H. Chen, "Music emotion classification: a fuzzy approach", Proceedings of the 14th annual ACM international conference on Multimedia, ACM, (2006), pp. 81-84.

[10] All music guide, [Online] http://www.allmusic.com/.

[11] I. R. I. Adjective Image Scale, http://iricolor.com 
[12] I. R. I Image Research Institute Inc., "Best Color Selection for Beautiful Design, Color Combination", YoungJin.com, Seoul Korea, (2003).

[13] http://www.do2learn.com/organizationtools/EmotionsColorWheel/

\section{Authors}
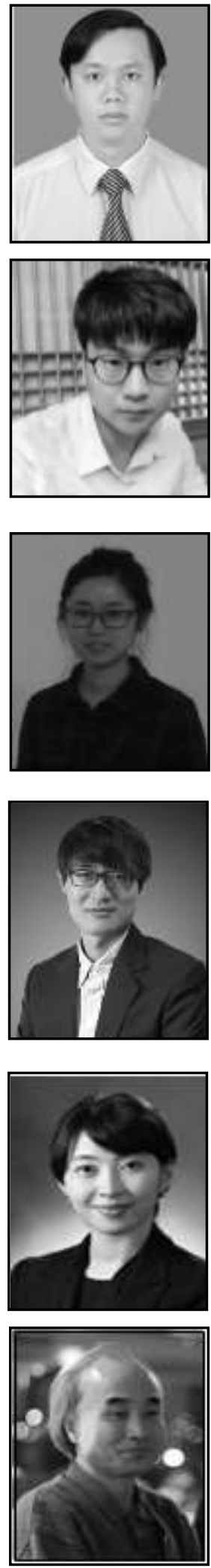

Van Loi Nguyen, he received his Master of Engineering in Computer Science from the University of Danang, Vietnam in 2010. $\mathrm{He}$ is currently a Ph.D. candidate at Soongsil University, Korea. His research interests include multimedia, information retrieval, database, and software testing.

Xu Bin, he received a Master degree in Media Engineering from Soongsil University in 2016. His research interests include database, multimedia, and mobile \& web programming.

Li Hui, she received a Master degree in Media Engineering from Soongsil University in 2015. Her research interests include database, multimedia, and web programming.

Lin Bin, he received a Master degree in Media Engineering from Soongsil University in 2014 . He is currently a Ph.D. candidate at Soongsil University. His research interests include database, SNS, and mobile \& web programming.

Donglim Kim, she received a Master degree and a Ph.D. degree in Media Engineering from Soongsil University in 2011 and 2016 respectively. Her research interests include multimedia, motion engineering, content engineering.

Younghwan Lim, he received his Master from the Dept. of Computer Science of Korea Advanced Institute of Science \& Technology in 1979, a Ph.D. degree from Northwestern University in 1985. From 1996 to the present, he has been a professor in Global School of Media, Soongsil University, Korea. His research interests include mobile solutions, multimedia, and creative engineering design. 
International Journal of Multimedia and Ubiquitous Engineering

Vol.12, No.1 (2017) 